\title{
ESTUDIO TEÓRICO DE LAS PROPIEDADES ÓPTICAS NO LINEALES EN ESPECIES 4-AMINO-4'-NITRO DIFENILSULFURO-3-SUSTITUIDO
}

\author{
Jean P. Incháustegui ${ }^{1}$, Rodolfo Pumachagua ${ }^{1 *}$
}

\begin{abstract}
RESUMEN
En este trabajo se determina las propiedades estructurales y ópticas de especies 4-amino-4'nitrodifenilsulfuro, tales como: momento dipolar estático (ì), polarizabilidad media $\left(a_{0}\right)$, anisotropía de la polarizabilidad (Äá) y la primera hiperpolarizabilidad promedio $\left(\hat{\mathrm{a}}_{0}\right)$. Los datos fueron obtenidos mediante la Teoría del Funcional de densidad, empleando el funcional híbrido B3LYPy la función base 6-31G.

Palabras clave: Óptica no lineal, momento dipolar, polarizabilidad, hiperpolarizabilidad, difenil sulfuro.

\section{THEORETICAL STUDY OF NONLINEAR OPTICAL PROPERTIES IN 3-SUBSTITUTED-4-AMINO-4'-NITRO DIPHENYLSULFIDE}

\begin{abstract}
This work identifies the structural and optical properties of species 4-amino-4'nitrodiphenilsulfide such as: static dipole moment (ì), mean polarizability $\left(a_{0}\right)$, anisotropy of the polarizability ( ̈̈á) and the first hyperpolarizability average $\left(\hat{\mathrm{a}}_{0}\right)$. Data were obtained through the theory of the density functional, using B3LYP hybrid functional and feature 6$31 \mathrm{G}$ basis.
\end{abstract}

Key words: Nonlinear optics, dipolar moment, polarizability, hyperpolarizability, diphenyl sulfide.

\section{INTRODUCCIÓN}

La óptica no lineal se ha desarrollado en los últimos años como un importante campo de investigación debido a su potencial aplicabilidad en el área de la fotoelectrónica y en un futuro inmediato a la tecnología fotónica. En las últimas décadas muchas moléculas orgánicas han mostrado respuestas ópticas no lineales extremadamente largas ${ }^{1}$ en comparación con cristales inorgánicos tradicionales; los materiales orgánicos tienen propiedades superiores tales como tiempos de respuesta ultrarrápidos, bajas constantes dieléctricas y técnicas sintéticas químicas flexibles.

Las propiedades ópticas no lineales están determinadas por la primera y segunda hiperpolarizabilidad; para tener los mejores valores se debe conocer detalladamente la estructura electrónica molecular.

Un compuesto orgánico con una posible respuesta óptica no lineal de segundo orden será un compuesto constituido por un fragmento dador y otro aceptor de electrones unidos covalentemente a través de un espaciador, el cual debe contener un sistema de electrones

\footnotetext{
Universidad Nacional Federico Villarreal, Facultad de Ciencias Naturales y Matemáticas, Laboratorio de Química Teórica, Jr. Chepen s/n (El Agustino) Perú. pumachagua@yahoo.es
} 
$\pi$ deslocalizado $^{2}$. La estructura del 4-amino-4'-nitrodifenilsulfuro se ha evidenciado por tener alto valor de intensidad de generación de segundo armónico ${ }^{3,4}$.

La descripción teórica más aceptada para explicar el fenómeno de óptica no lineal de segundo orden, en moléculas dipolares conjugadas, consiste en el modelo de alternancia en la longitud de onda de enlace propuesta por Marder ${ }^{5}$. Este modelo correlaciona el coeficiente â con el grado de polarización o separación de cargas en el estado fundamental. Este grado de polarización dependerá básicamente de la estructura química del compuesto a estudiar.

El objetivo es evaluar las propiedades ópticas del 4-amino-4'-nitrodifenilsulfuro, conteniendo diferentes grupos en el carbono 3, dentro del formalismo de la teoría del funcional de la densidad (DFT).

\section{FUNDAMENTOS TEÓRICOS}

La teoría cuántica de la suma de todos los estados (SOS) en la respuesta óptica no lineal ${ }^{6}$ postula que la susceptibilidad no lineal de un sistema cuántico comienza con el cálculo del momento dipolar inducido como una función del campo eléctrico expandido como una serie de Taylor. El momento dipolar es dado usando la función de onda del estado fundamental de una molécula que incluye acoplar el campo eléctrico aplicado.

El m-ésimo estado de energía de una molécula en presencia de un campo eléctrico local está dado por $\left|\psi_{m}(F)\right\rangle$, donde:

$$
\vec{F}(t)=\sum_{p=1}^{\text {no. incident fields }} \vec{F}_{p}\left(\omega_{p}, t\right)
$$

A 0K, el operador polarización es dado por:

$$
\vec{P}(\vec{F})=\left\langle\psi_{g}(\vec{F}(t))|\vec{P}| \psi_{g}(\vec{F}(t))\right\rangle
$$

donde $\left|\psi_{g}[\vec{F}(t)]\right\rangle$ es el estado fundamental perturbado; la usceptibilidad molecular es dada por:

$$
\xi_{i j k \ldots \ell}^{(n)}\left(-\omega ; \omega_{1}, \omega_{2}, \omega_{n}\right)=\left.\frac{1}{\varepsilon_{0} D^{\prime}} \frac{\partial^{n}}{\partial F_{j}\left(\omega_{1}\right) \partial F_{k}\left(\omega_{2}\right) \ldots \partial F_{\ell}\left(\omega_{n}\right)}\left\langle\psi_{g}(\vec{F})\left|P_{i}\right| \psi_{g}(\vec{F})\right\rangle\right|_{\vec{F}=0}
$$

donde $\mathrm{D}^{\prime}$ es el denominador de degeneración dependiente de la frecuencia que varía de acuerdo al número de frecuencias distintas y número de campos a frecuencia 0 .

Cuando el campo eléctrico de la luz es mucho más débil que los campos eléctricos que mantienen unida a la molécula, la función de onda molecular puede ser determinada usando la teoría de perturbaciones. Se define $\mathrm{H}_{0}$ como el Hamiltoniano sin perturbar dado por:

$$
i \hbar \frac{\partial}{\partial t}|\psi\rangle=H_{0}|\psi\rangle
$$

El potencial de perturbación dependiente del tiempo es dado por:

$$
V(t)=-\sum \vec{\mu} \cdot \vec{F}_{p}(t)
$$


donde $\vec{\mu}$ es el momento dipolar molecular y ñ abarca todos los campos fotónicos. La función de onda evolucionará así:

$$
\left|\psi_{g}(t)\right\rangle=\left|\psi_{g}^{(0)}\right\rangle+\lambda \sum_{m} a_{m}^{(1)}(t)\left|\psi_{m}^{(0)}\right\rangle e^{i \hat{\omega}_{m g} t}+\lambda^{2} \sum_{v} a_{v}^{(2)}(t)\left|\psi_{v}^{(0)}\right\rangle e^{i \hat{\omega}_{\mathrm{kg}} t}+\cdots
$$

Dónde:

$$
\left|\psi_{m}^{(0)}(t)\right\rangle=\left|\psi_{m}^{(0)}\right\rangle e^{-i \hat{\omega}_{m g} t}
$$

El momento dipolar inducido en $\lambda$ es:

$$
\langle\vec{\mu}\rangle^{(1)}(t)=\sum_{m} a_{m}^{(1) *}(t)\left\langle\psi_{g}^{(0)}\left|\vec{\mu} e^{i \hat{\omega}_{m g}^{*} t}\right| \psi_{m}^{(0)}\right\rangle+\left\langle\psi_{m}^{(0)}\left|\vec{\mu} \sum_{m} a_{m}^{(1)}(t)\right| \psi_{g}^{(0)}\right\rangle e^{-i \hat{\omega}_{m g} t}
$$

La polarizabilidad lineal molecular es dada por:

$$
\alpha_{i j}^{(1)}\left(-\omega_{p} ; \omega_{p}\right)=\frac{1}{\varepsilon_{0} \hbar} L\left(\omega_{p}\right)\left(\frac{\mu_{g m, i} \mu_{m g, j}}{\hat{\omega}_{m g}^{*}+\omega_{p}}+\frac{\mu_{g m, j} \mu_{m g, i}}{\hat{\omega}_{m g}-\omega_{p}}\right)
$$

La hiperpolarizabilidad es dada por:

$$
\begin{aligned}
& \beta_{i j k}\left(-\left[\omega_{p} \pm \omega_{q}\right] ; \omega_{p}, \pm \omega_{q}\right)=\frac{1}{\hbar^{2} \varepsilon_{0}} L\left(\omega_{p} \pm \omega_{q}\right) L\left(\omega_{p}\right) L\left( \pm \omega_{q}\right) \\
& \times \sum_{n m}\left\{\frac{\mu_{g n, i} \mu_{n m, k} \bar{\mu}_{m g, j}}{\left(\hat{\hat{\omega}}_{n g} \mp \omega_{q}-\omega_{p}\right)\left(\hat{\hat{\omega}}_{m g}-\omega_{p}\right)}+\frac{\mu_{g n, k} \mu_{n m, i} \bar{\mu}_{m g, j}}{\left(\hat{\hat{\omega}}_{n g}^{*} \pm \omega_{q}\right)\left(\hat{\hat{\omega}}_{m g}-\omega_{p}\right)}\right. \\
+ & \left.\frac{\mu_{n m, j} \mu_{g n, k} \bar{\mu}_{m g, j}}{\left(\hat{\hat{\omega}}_{m g}^{*} \pm \omega_{q}+\omega_{p}\right)\left(\hat{\hat{\omega}}_{n g}^{*}+\omega_{q}\right)}\right\}
\end{aligned}
$$

\section{ASPECTOS COMPUTACIONALES}

Los cálculos fueron llevados a cabo con el programa Gaussian 09. Los cálculos DFT fueron realizados con el funcional B3LYP y la función base 6-31G debido a los buenos resultados que éste produce y el bajo costo computacional ${ }^{7,8}$. Las optimizaciones de geometría se realizaron mediante el método por gradiente analítica de Berny.

Los parámetros ópticos tales como la primera hiperpolarizabilidad promedio $\left(\hat{\mathrm{a}}_{0}\right)$, la polarizabilidad media $\left(a_{0}\right)$ y el momento dipolar (î) han sido calculados usando el funcional y la función base de las optimizaciones; éstos se calculan directamente:

$$
\begin{gathered}
\mu=\mu_{x}{ }^{2}+\mu_{y}{ }^{2}+\mu_{z}{ }^{2} \\
\alpha_{0}=\frac{\alpha_{x x}+\alpha_{y y}+\alpha_{z z}}{3}
\end{gathered}
$$




$$
\begin{aligned}
& \Delta \alpha=2^{\frac{1}{2}}\left[\left(\alpha_{x x}-\alpha_{y y}\right)^{2}+\left(\alpha_{y y}-\alpha_{x x}\right)^{2}+6 \alpha_{x x}^{2}\right]^{\frac{1}{2}} \\
& \beta=\left(\beta_{x}{ }^{2}+\beta_{y}{ }^{2}+\beta_{z}{ }^{2}\right)^{\frac{1}{2}} \\
& \beta_{x}=\beta_{x x x}+\beta_{x y y}+\beta_{x z z} \\
& \beta_{y}=\beta_{y y y}+\beta_{x x y}+\beta_{y z z} \\
& \beta_{z}=\beta_{z z z}+\beta_{x x z}+\beta_{y y z}
\end{aligned}
$$

En presencia de un campo eléctrico aplicado, la energía de un sistema es una función del campo eléctrico y la primera hiperpolarizabilidad como un tensor de tercer orden que puede ser descrito como una matriz $3 \times 3 \times 3$. Los 27 componentes de esa matriz de 3 dimensiones pueden ser reducidos a 10 componentes por la simetría de Kleinman.

Cuando el campo eléctrico es débil y homogéneo se define como:

$$
U(E)=U^{0}-\sum_{i} \mu^{0} E_{i}-\frac{1}{2} \sum_{i j} \alpha_{i j} E_{i} E_{j}-\frac{1}{3} \times \sum_{i j k} \beta_{i j k} E_{i} E_{j} E_{k}-\frac{1}{4} \sum_{i j k l} \gamma_{i j k l} E_{i} E_{j} E_{k} E_{l}
$$

(XVIII)

donde $\mathrm{E}^{0}$ es la energía de la molécula sin perturbar, $\mathrm{E}_{\mathrm{a}}$ es el campo de origen y ì, á, â y ã son los componentes del momento dipolar, polarizabilidad, primera hiperpolarizabilidad y segunda hiperpolarizabilidad respectivamente, éstas son calculadas de la siguiente manera:

$$
\begin{aligned}
\alpha_{i j} & =\left.\frac{\partial U^{2}(E)}{\partial E_{i} \partial E_{j}}\right|_{E=0} \\
\beta_{i j k} & =\left.\frac{\partial^{3} U(E)}{\partial E_{i} \partial E_{j} \partial E_{k}}\right|_{E=0} \\
\gamma_{i j k l} & =\left.\frac{\partial^{4} U(E)}{\partial E_{i} \partial E_{j} \partial E_{k} \partial E_{L}}\right|_{E=0}
\end{aligned}
$$

\section{RESULTADOS Y DISCUSIÓN}

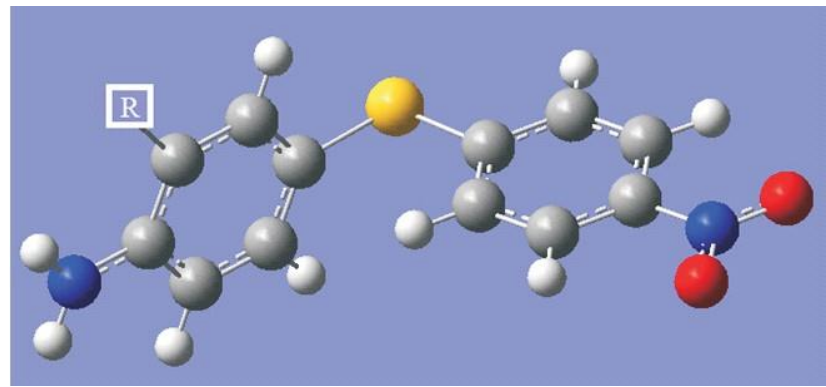

Figura 1. 4-Amino-4'-nitrodifenilsulfuro 
Tabla 1. Nomenclatura IUPAC

\begin{tabular}{cc}
\hline 4-Amino-4'-nitrodifenilsulfuro...(a) & 4-Amino-3-metoxi-4'-nitrodifenil \\
& sulfuro...(f) \\
3,4-Diamino-4'-nitrodifenilsulfuro...(b) & 4-Amino-3-cloro-4'-nitrodifenil \\
& sulfuro...(g) \\
& 4-Amino-3,4'-dinitrodifenilsulfuro...(h) \\
4-Amino-3-hidroxi-4'-nitrodifenil & \\
sulfuro............(c) & 4-Amino-3-ciano-4'-nitrodifenil \\
4-Amino-3-metil-4'-nitrodifenil & sulfuro...(i) \\
sulfuro............(d) & 4-Amino-3-bromo-4'-nitrodifenil \\
4-Amino-3-fenil-4'-nitrodifenil & sulfuro...(j) \\
sulfuro..........(e) &
\end{tabular}

La figura 1 muestra al sistema molecular base; contiene un grupo aceptor: nitro, otro dador: amino y un espaciador: difenilsulfuro. Los grupos dador-aceptor generan un estado con separación de cargas, incrementando las propiedades ópticas no lineales; cualquier variación en los grupos, dador-aceptor modificará la distribución de la forma resonante con separación de cargas, favoreciendo al fenómeno de óptica no lineal de segundo orden.

En el sistema: 4-amino-4'-nitrodifenilsulfuro, la sustitución en el carbono-3 por diferentes grupos permitirá mejorar la topología del sistema espaciador ð-conjugado entre ambos fragmentos dador-aceptor, como se observa en la tabla 1.

Tabla 2. Valores de momento dipolar

\begin{tabular}{ccccc}
\hline Sistema & $\boldsymbol{\mu}_{\mathbf{x}}$ & $\boldsymbol{\mu}_{\mathbf{y}}$ & $\boldsymbol{\mu}_{\mathrm{z}}$ & $\boldsymbol{\mu}($ Debye $)$ \\
$\mathbf{a}$ & $-8,4629$ & $-0,5526$ & 0,0003 & 8,4809 \\
$\mathbf{b}$ & 8,5072 & $-0,3935$ & 0,6438 & 8,5406 \\
$\mathbf{c}$ & 7,4152 & 0,4276 & 0,4567 & 7,4416 \\
$\mathbf{d}$ & 8,6659 & $-0,3747$ & $-0,2569$ & 8,6778 \\
$\mathbf{e}$ & 8,2949 & $-2,2741$ & 0,9417 & 8,6523 \\
$\mathbf{f}$ & 7,8041 & 0,1930 & 0,5403 & 7,8251 \\
$\mathbf{g}$ & $-6,9102$ & 1,2260 & 2,5386 & 7,4631 \\
$\mathbf{h}$ & $-4,4360$ & 2,6445 & 5,2326 & 7,3250 \\
$\mathbf{i}$ & $-5,4618$ & 2,0434 & 4,1904 & 7,1809 \\
$\mathbf{j}$ & $-6,8845$ & 2,1559 & 2,1454 & 7,5264 \\
\hline
\end{tabular}

Tabla 3.Valores de polarizabilidad

\begin{tabular}{lccccccccc}
\hline tema & $\alpha_{\mathrm{xx}}$ & $\alpha_{\mathrm{xy}}$ & $\alpha_{\mathrm{yy}}$ & $\alpha_{\mathrm{xz}}$ & $\alpha_{\mathrm{yz}}$ & $\alpha_{\mathrm{zz}}$ & $\alpha_{0}$ (a.u.) & $\Delta$ á & $\alpha_{0}$ (e.s.u.) \\
& & & & & & & & & x $10^{-12}$ \\
$\mathbf{a}$ & 264,524 & 20,186 & 155,552 & 0,020 & 0,003 & 99,344 & 173,14 & 941,891 & 25,659 \\
$\mathbf{b}$ & 274,068 & $-18,390$ & 158,923 & $-5,408$ & 4,118 & 115,022 & 182,671 & 976,921 & 27,072 \\
$\mathbf{c}$ & 268,802 & $-18,264$ & 155,628 & $-9,378$ & 5,926 & 109,555 & 177,995 & 958,264 & 26,379 \\
$\mathbf{d}$ & 277,748 & $-16,915$ & 162,931 & $-6,386$ & 5,208 & 115,660 & 185,446 & 962,165 & 27,483 \\
e & 322,486 & 10,760 & 195,861 & 10,808 & 16,704 & 195,961 & 238,103 & 1145,459 & 35,286 \\
$\mathbf{f}$ & 278,086 & $-10,842$ & 159,076 & $-18,157$ & 12,742 & 134,223 & 190,462 & 992,278 & 28,226 \\
$\mathbf{g}$ & 274,870 & 17,460 & 155,717 & $-10,723$ & $-8,248$ & 120,786 & 183,791 & 981,467 & 27,238 \\
$\mathbf{h}$ & 283,156 & 20,817 & 155,842 & $-8,300$ & $-9,212$ & 137,680 & 192,226 & 1013,445 & 28,488 \\
$\mathbf{i}$ & 281,015 & 22,915 & 159,696 & 0,341 & $-3,281$ & 127,012 & 189,241 & 1003,238 & 28,046 \\
j & 279,971 & 7,073 & 149,970 & $-20,777$ & $-13,163$ & 133,795 & 187,912 & 1004,085 & 27,849 \\
\hline
\end{tabular}


Tabla 4. Valores de hiperpolarizabilidad

\begin{tabular}{cccccc}
\hline Sistema & $\beta_{\mathrm{xxx}}$ & $\beta_{\mathrm{yyy}}$ & $\beta_{\mathrm{zzz}}$ & $\beta_{0}$ (a.u.) & $\beta_{0}$ (e.s.u.) $\times 10^{-30}$ \\
$\mathbf{a}$ & 355,619 & 299,302 & 457,253 & 1066,910 & 9,217 \\
$\mathbf{b}$ & $-371,301$ & $-411,020$ & $-125,441$ & 1294,645 & 11,184 \\
$\mathbf{c}$ & $-359,186$ & $-988,091$ & $-747,444$ & 1084,690 & 9,370 \\
$\mathbf{d}$ & $-353,109$ & $-707,144$ & $-342,149$ & 448,655 & 3,876 \\
$\mathbf{e}$ & $-298,469$ & 420,453 & $-595,478$ & 1289,166 & 11,137 \\
$\mathbf{f}$ & $-358,396$ & $-850,806$ & $-365,174$ & 1693,952 & 14,635 \\
$\mathbf{g}$ & 321,409 & $-926,120$ & 116,628 & 1054,257 & 9,108 \\
$\mathbf{h}$ & 268,519 & $-132,175$ & $-147,496$ & 1670,268 & 14,430 \\
$\mathbf{i}$ & 309,163 & $-709,678$ & 107,209 & 1752,856 & 15,143 \\
$\mathbf{j}$ & 307,387 & $-205,671$ & 655,057 & 2499,567 & 21,595 \\
\hline
\end{tabular}

La búsqueda de sistemas con interesantes propiedades ópticas no lineales requiere la optimización de los coeficientes microscópicos de las moléculas aisladas como la hiperpolarizabilidad $\beta_{0}$.

La tabla 2 muestra los valores de momento dipolar $\mu$ para los sistemas estudiados, en su estado fundamental. La molécula base a presenta $\mu=8,4809$ Debye. Las moléculas: b, $\mathbf{d}$ y e contienen a los grupos: amino, metilo y fenilo respectivamente, las cuales presentan un ligero incrementodevaloresì.

La tabla 3 muestra los valores de polarizabilidad á para todos los sistemas. La molécula base a presenta $\alpha_{0}=25,659 \times 10^{-12}$ e.s.u. Todas las demás moléculas presentan valores mayores.

La tabla 4 muestra los valores de hiperpolarizabilidad de primer orden $\beta_{0}$, responsable de los efectos ópticos no lineales de segundo orden. La molécula base a, presenta $\hat{a}_{0}=9,217 \times 10^{-30}$ e.s.u. Los sistemas con grupo sustituido en el carbono-3 de acuerdo con los valores de la tabla 4 pueden discutirse en tres grupos diferentes.

En primer lugar, las moléculas: c, d y g que contienen los grupos: hidroxilo, metilo y cloruro respectivamente, presentan los valores más bajos de hiperpolarizabilidad. Los grupos: hidroxilo y metilo son activantes del sistema ð-conjugado y el cloruro es desactivante débil.

En segundo lugar, los sistemas: b, e y f contienen los grupos: amino, fenilo y metoxilo respectivamente, éstas presentan valores ligeramente mayores de hiperpolarizabilidad. Estos grupos son activantes del sistema aromático.

En tercer lugar, los sistemas: h, i y j contienen los grupos: nitro, ciano y bromo, respectivamente, presentan los mayores valores de hiperpolarizabilidad. Estos grupos son desactivantes del sistema aromático que tendrán mayor impacto en la producción de la generación de segundo armónico.

\section{CONCLUSIONES}

El modelo de la teoría funcional de densidad: B3LYP/6-31G describe adecuadamente las propiedades ópticas no lineales en los sistemas derivados del 4-amino-4 nitrodifenilsulfuro. La sustitución en el carbono-3 de grupos desactivantes al sistema espaciador ð-aromático incrementa los valores de hiperpolarizabilidad, favoreciendo el fenómeno óptico no lineal de segundo orden.

\section{AGRADECIMIENTO}

Los autores agradecen al Dr. William Tiznado Vásquez de la Universidad Andrés Bello, Santiago de Chile, por brindarnos soporte computacional. 


\section{REFERENCIAS}

1. María del Carmen Ruiz Delgado, Oligotiofenos funcionalizados simétrica o asimétricamente con aplicaciones en optoelectrónica: Estudio espectroscópico y teórico. Tesis Doctoral, Departamento de Química Física, Facultad de Ciencias, Universidad de Málaga, 2006.

2. J. L. Oudar \& D. S. Chemla, Hyperpolarizabilities of the nitroanilines and their relations to the excited state dipole moment. J. Chem. Phys., 1977; 66: 2664-2668.

3. Hamzed M. Abdel-Halim, Second-order non-linear optical properties of some organic compounds in powder. Indian Journal of Engineering and Materials Sciences, 2004; 11 : 207-211.

4. Seth R. Marder, John E. Sohn \& Galen D. Stucky (editors), Materials for nonlinear optical: Chemical perspectives. ACS Symposium Series 455, American Chemical Society, Washington, DC. 1991.

5. S. R. Marder, D. N. Beratan \& L. -T. Cheng, Approaches for optimizing the first electronic hyperpolarizability of conjugated organic molecules. Science, 1991; 252: 103106.

6. Mark G. Kuzyk, Kenneth D. Singer \& George I. Stegeman, Theory of molecular nonlinear optics, Advances in Optics and Photonics, 2013; 5: 4-82.

7. Gyoosoon Park, Woo Sik Jung \& Choon Sup Ra, First Hyperpolarizabilities of nonlinear optical compounds: Susceptibility in donor-acceptor stilbene analogs. Bull. Korean Chem. Soc., 2004; 25: 1427-1429.

8. Tsung-Yi Lin, Ajay Chaudhari \& Shyi-Long Lee, Correlation between substituent constants and hyperpolarizabilities for di-substituted trans-azobenzenes. J. Mol. Model., 2013; 19:529-538. 